\title{
Intravenous leiomyomatosis with intracardiac extension depicted on computed tomography and magnetic resonance imaging scans: A report of two cases and a review of the literature
}

\author{
HANJIANG ZENG, ZHONGZI XU, LIZHI ZHANG, YI LUO, HUI CHEN, \\ HONGJI ZHU, LIQING PENG and JIANQUN YU \\ Department of Radiology, West China Hospital, Sichuan University, Chengdu, Sichuan 610041, P.R. China
}

Received February 4, 2015; Accepted March 15, 2016

DOI: $10.3892 / \mathrm{ol} .2016 .4499$

\begin{abstract}
Intravenous leiomyomatosis (IVL) is defined as a benign smooth muscle tumor, growing within systemic veins. IVL with intracaval and intracardiac extension has rarely been reported in radiological and oncological journals. The present study describes 2 cases of IVL extending from the inferior vena cava to the right atrium and ventricle, and discusses the imaging findings and differential diagnosis of this tumor entity. The two patients, who complained of palpitations, shortness of breath or syncope, were surgically treated, with complete resection of the cardiac and intracaval tumors. Pathological examinations were suggestive of IVL. The postoperative course of the two patients was uneventful, and no signs of recurrence were observed on follow-up. Computed tomography and magnetic resonance imaging played a vital role in the diagnostic process and presurgical assessment. The results of the present study indicate that IVL should be considered upon presentation of a soft mass in systemic veins, even when the mass extends to the right cardiac chambers, in female patients, particularly in patients with a history of uterine myoma.
\end{abstract}

\section{Introduction}

Intravenous leiomyomatosis (IVL) is a rare, histologically benign, smooth muscle tumor. It is typically associated with a mesenchymal tumor of the uterus with macroscopic intravascular proliferation (1). Although IVL is histologically benign, it may occasionally exhibit malignant behavior, due to its growth along the pelvic veins and the inferior vena cava (IVC), extending into the cardiac chambers and pulmonary vasculature (2). Due to its rarity and atypical clinical features,

Correspondence to: Professor Jianqun Yu, Department of Radiology, West China Hospital, Sichuan University, 37 Guo Xue Xiang, Chengdu, Sichuan 610041, P.R. China

E-mail: cjr.yujianqun@vip.163.com

Key words: intravenous leiomyomatosis, intracardiac tumors, computed tomography, magnetic resonance imaging the condition may be misdiagnosed as a primary cardiac tumor or a venous thrombus, which may lead to potentially life threatening consequences. Hence, early and accurate diagnosis and appropriate treatment choices are important for the patient prognosis. Complete surgical resection (single or staged procedures) is the treatment option for IVL, as it is key to preventing recurrence (3). The present study reports 2 cases of IVL that were diagnosed using dual-source computed tomography (CT) and magnetic resonance imaging (MRI), as well as clinical and pathological examinations. A review of the English literature on IVL was also performed.

\section{Case report}

Case 1. A 46-year-old female patient was referred to the West China Hospital of Sichuan University (Chengdu, China) in December 2013, presenting with a 10-day history of palpitations and shortness of breath that were exacerbated upon exertion. Physical examination revealed slight bilateral leg edema and no other abnormalities. Echocardiography showed a mobile, solid mass in the right atrium that originated from the IVC. The lesion, which almost blocked the tricuspid valve, was initially diagnosed as atrial myxoma or thrombus. The serum levels of the tumor marker carbohydrate antigen (CA) 125 were mildly elevated (41.93 U/ml; normal value, $<35 \mathrm{U} / \mathrm{ml}$ ), while all other blood and biochemical markers were within the normal ranges. The patient's medical history included a myomectomy (on October 2000), with no notable family history.

Thoracic and abdominal CT scans (Somatom Definition Flash; Siemens AG, Munich, Germany) were performed with and without contrast media. On the CT scans, a filling defect was observed in the IVC and right atrium, with a soft tissue density of 20-22 HU on plain images and 28-62 HU on contrast-enhanced images. The lesion stretched into the right ventricle through the tricuspid valve, and stretched as far as the left common iliac vein and internal iliac vein inferiorly. In addition, an ill-defined mass with heterogeneous attenuation was detected in the pelvic region; the mass originated from the uterus and involved the left gonadal vein, resulting in luminal stenosis. The CT value of the pelvic lesion was 15-31 HU on plain images and 31-52 HU on contrast-enhanced images. The lesion in the right atrium and IVC appeared to be 
anatomically associated with the mass located in the pelvic region (Fig. 1). Furthermore, tortuous collateral veins were observed around the IVC. Cardiac cine MRI (Magnetom Trio Tim; Siemens AG) showed that the circumscribed mass moved in parallel with the cardiac motion in the right cardiac cavities. Gadolinium-enhanced MRI of the heart revealed a large streak-shaped filling defect in the IVC and right atrium that was mildly hyperintense on T1- and T2-weighted imaging (WI), and heterogeneously enhanced following contrast-enhanced scanning (Fig. 2).

Considering the imaging findings, complete surgical excision of the tumor in the IVC was performed carefully through a right atriotomy and longitudinal venotomy in January 2014. The tumor had no stalk or obvious adhesion with the wall of the right atrium.

The resected gross intracaval specimen was a yellowish-white tumor with a smooth border and a tip. The tumor appeared as a sausage-shaped protrusion with a maximum diameter of $20 \mathrm{~cm}$. Immunohistochemical analysis using monoclonal antibodies revealed positivity for the smooth muscle markers desmin (Biocare Medical LLC, Concord, CA, USA) and smooth muscle actin (SMA; OriGene Technologies Inc., Rockville, MD, USA), and negativity for h-caldesmon (Epitomics, Burlingame, CA, USA), human melanoma black 45 (HMB45) and S-100 (both Leica Microsystems Inc., Buffalo Grove, IL, USA). Upon microscopy (CX31-72C02 microscope; Olympus, Corporation, Tokyo, Japan), the hematoxylin-eosin staining of tumor sections revealed smooth muscle cell proliferation without nuclear atypia or mitotic index. The MIB-1/Ki-67 (OriGene Technologies Inc.) labeling index was $\sim 2 \%$, confirming the non-malignant nature of the tumor. In addition, estrogen and progesterone (Epitomics) expression was detected in the nucleus (3).

The postoperative course of the patient was uneventful, and no signs of recurrence were observed at the 1-year follow-up examination in February 2015.

Case 2. A 45-year-old female patient presented to the West China Hospital of Sichuan University in November 2014 with a 4-month history of persistent palpitations and occasional syncope. The patient's past medical history consisted of a myomectomy on June 2007, with nothing of particular note in the family medical history. Upon physical examination, a diastolic murmur was auscultated in the tricuspid region. Laboratory test results were normal, with the exception of an increased level of CA 125 (56.93 U/ml). Echocardiography demonstrated a hypoechogenic mass in the right atrium and ventricle, suggesting a diagnosis of thrombi.

Contrast-enhanced CT scans performed from the chest to the pelvis revealed unilateral heterogeneous uterine masses in the right iliac vein and IVC that were indicative of multiple leiomyoma. The mass that caused almost complete occlusion of the IVC demonstrated enhancement similar to that of the uterine leiomyoma (Fig. 3). MRI of the chest and upper abdomen revealed a cord-like mass extending from the IVC into the right cardiac chambers and almost completely occupying the IVC lumen. The tumor exhibited low to intermediate signal intensity on T1WI and heterogeneous high signal intensity on T2WI, with mild enhancement observed in the late phase. The top of the mass extended from the right atrium into the right ventricle at the diastolic phase (Fig. 4). Based on these findings, the patient was diagnosed with IVL of the uterus with intravascular and cardiac extension.

Total abdominal hysterectomy, bilateral salpingo-oophorectomy, and intracardiac and intravenous mass excision were performed as a two-stage procedure under cardiopulmonary bypass in April 2013 and July 2013. Gross examination of the intravascular specimen revealed a smooth, wormlike mass, $3-4 \mathrm{~cm}$ in circumference and $26 \mathrm{~cm}$ in length, with a bulbous end corresponding to the intracardiac portion that almost blocked the tricuspid valve.

The excised tumor had a white cut surface, and was rubbery and firm on palpation. Immunohistochemical analysis of the IVL cells indicated positive staining for the smooth muscle markers desmin (Biocare Medical LLC) and SMA (OriGene Technologies Inc.), and negative staining for Ki-67 (OriGene Technologies Inc.), HMB45, S-100 (both Leica Microsystems Inc.), h-caldesmon (Epitomics) and p53 (OriGene Technologies Inc.), confirming its smooth muscle nature without signs of malignancy. Nuclear estrogen and progesterone receptor expression was observed in the intravascular tumor and uterine leiomyoma. The postoperative course was uneventful. The patient continued to recover well, and at the 18-month follow-up, there was no recurrence of the symptoms or tumor, and the patient resumed normal activities thereafter.

\section{Discussion}

IVL, which was first described by Brich-Hirschfeld in 1896 (4), is a rare benign tumor that is histologically characterized by nodular masses of smooth muscle cells growing within systemic veins. It typically originates from the uterus and may involve ovarian veins. Occasionally, the tumor occurs in other intravascular locations, distant from the uterus. Durck reported the first case of IVL with intracardiac extension in 1907 (5). The tumors were described as nodular or spiral within pelvic veins and the IVC, or extending to the right cardiac chambers and bilateral pulmonary arteries $(2,6,7)$. Two major hypotheses have been established regarding the etiology of IVL $(1,8)$. The first hypothesis suggests that the tumor arises from the vein walls, while the second proposes that the uterine leiomyoma is a primary tumor with intravascular projections into an adjacent venous channel. Fukuyama et al (9) suggested that the tumor does not invade the vessel by breaking the venous wall, but rather stretches the vascular wall to extend along the vascular lumen, while covered in endothelium.

A review of the English literature included by PubMed between January 2000 and January 2015 was performed using the keywords 'intravenous leiomyomatosis' AND 'last 15 years'[PDat], and 52 cases of IVL with CT or MRI descriptions were identified, as indicated in Table I (2,6,7,10-46). The median age of the patients was 44.8 years old (age range, 20-70 years). The majority of cases were diagnosed intraoperatively. Common initial symptoms included pelvic pain or abnormal uterine bleeding; however, certain patients presented with chest pain, palpitation, shortness of breath, dyspnea on exertion, edema of the lower extremities or even sudden mortality. The clinical signs and symptoms predominantly depended on the degree of intravascular obstruction caused by the tumor. Heart failure may result in misdiagnosis or 

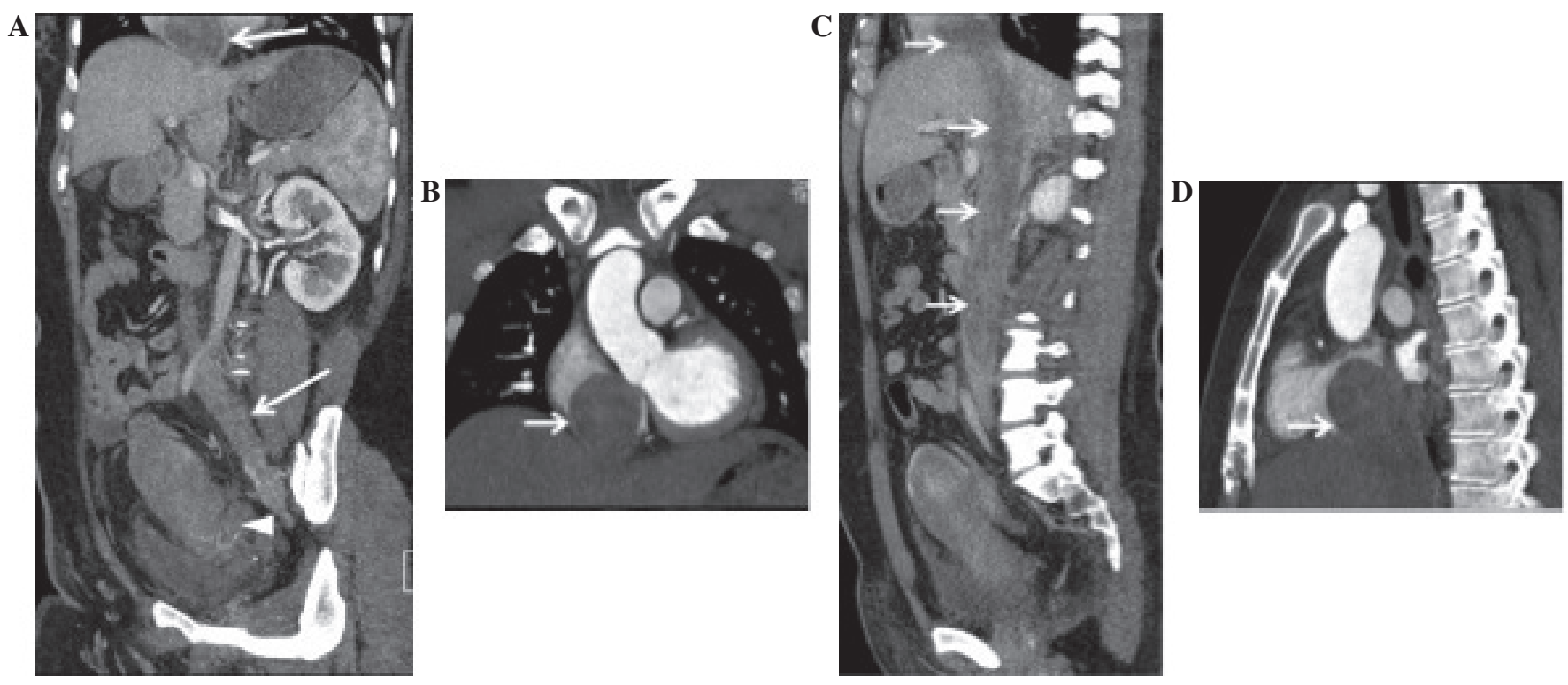

Figure 1. Case 1: Contrast-enhanced computed tomography scans. (A and B) Oblique-coronal and (C and D) sagittal reconstruction images showing a cord-like mass (arrows) in the lumen of the left iliac vein, extending to the inferior vena cava and right atrium. (A) The enhancement features of the intravenous mass were similar to those of the uterine mass (triangle).
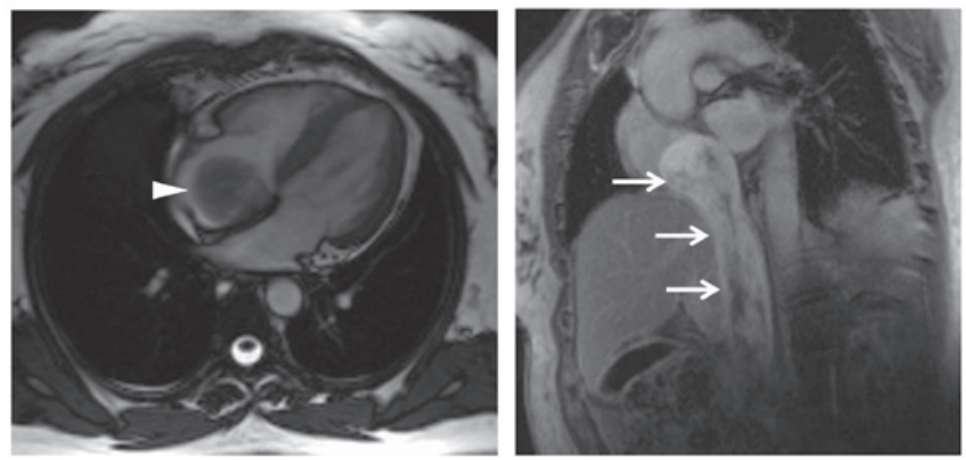

Figure 2. Case 1: Axial T2-weighted magnetic resonance imaging scan of the heart showing a mass with low signal intensity in the right atrium (triangle; left) and a lesion involving the inferior vena cava, which exhibited heterogeneous enhancement following contrast agent administration (arrows; right).
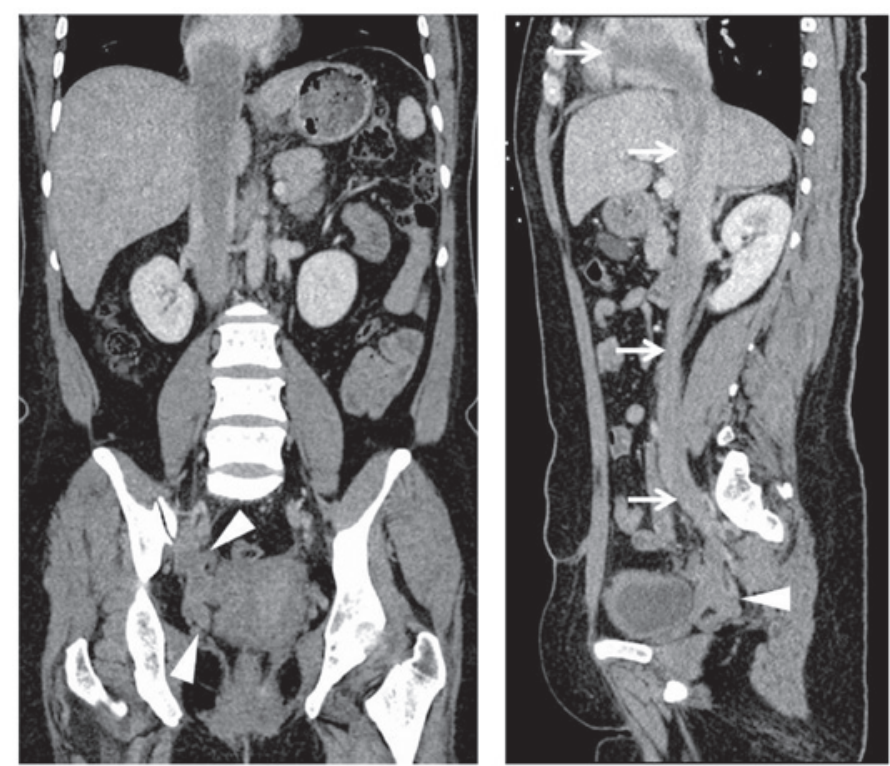

Figure 3. Case 2: Coronal (left) and sagittal (right) reconstruction images of the contrast-enhanced computed tomography scan showing that the uterine tumor had invaded the right parametrial veins and extended to the right atrium through the right common iliac vein (triangle) and inferior vena cava (arrows). The lesions exhibited an isodensity and enhancement similar to those of the adjacent hysteromyoma. 

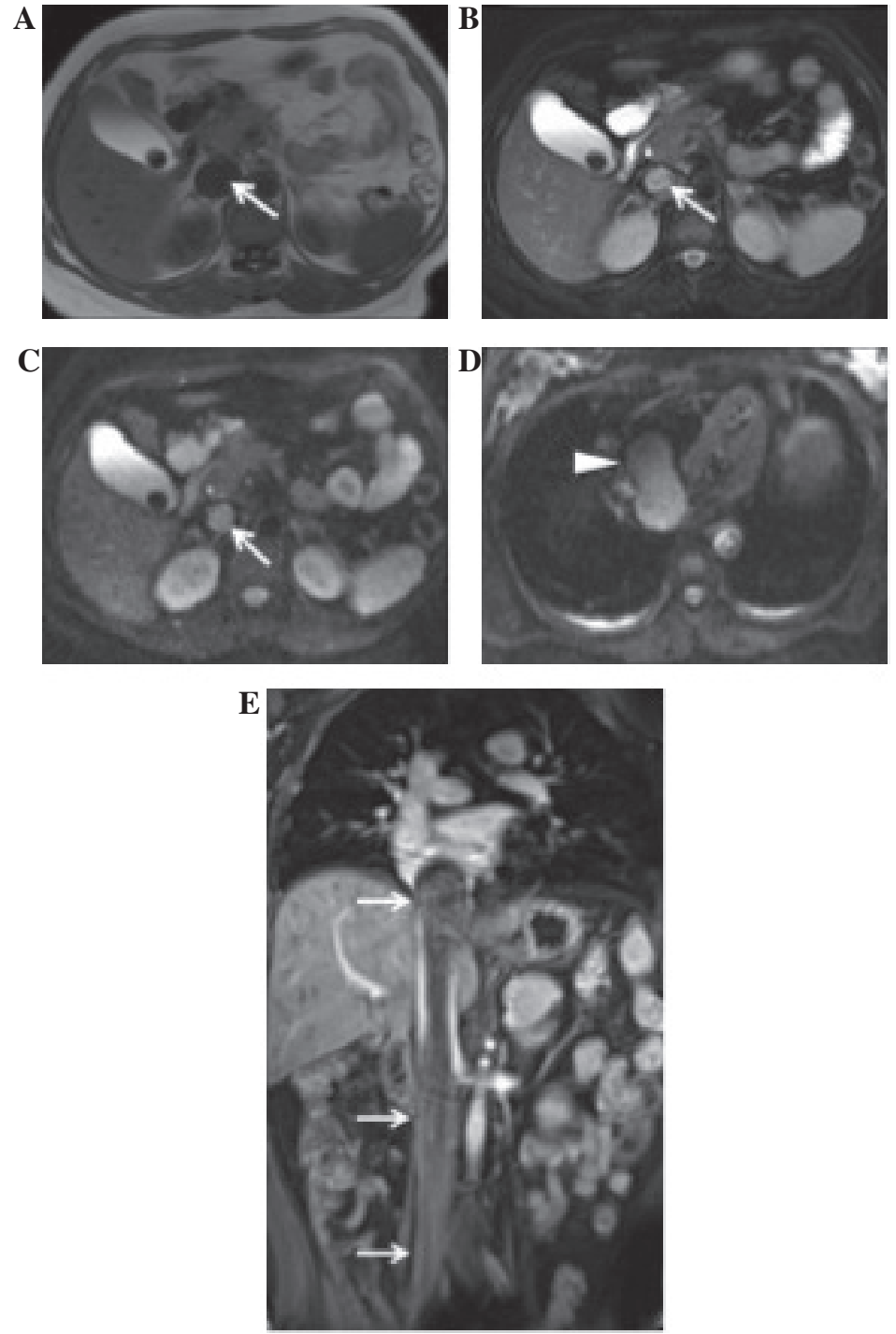

Figure 4. Case 2: Axial (A) T1- and (B) T2-weighted fast spin-echo magnetic resonance imaging (MRI) showing a polypoid mass within the inferior vena cava (IVC; arrow), with (C and D) restricted diffusion on diffusion-weighted MRI. (D) Cardiac MRI scan showed that the distal portion of the mass had moved into the right ventricle through the tricuspid valve during diastole (triangle). (E) Contrast-enhanced MRI revealed that the tubular, serpent-like filling defect (arrows) almost completely occupied the IVC.

delayed diagnosis of IVL until further examination or sudden mortality due to fatal intracardiac obstruction.

In the majority of the reviewed cases, the initial pathway of extension for IVL was unilateral through the common iliac veins, while certain cases exhibited IVL with intracardiac extension and concomitant uterine leiomyoma. Various unusual patterns of intravenous growth were observed in the 52 recorded tumors; the distal end of 11 tumors $(21.2 \%)$ were confined to the IVC, $30(57.7 \%)$ extended to right atrium, $8(15.4 \%)$ to the right ventricle and $3(5.8 \%)$ to the pulmonary arteries. Tumor mobility was recorded in 42 of these cases, however, due to the varying diameters of the tumors, adherence to the wall of the IVC or the right chambers were reported in 26 cases. The distal end of the tumor in the present cases extended intracardially to the right atrium and right ventricle. Both of these tumors moved in parallel with cardiac motion.

CT and MRI scans are the most useful imaging modalities for the diagnosis of IVL. The high-density resolution and multiplanar capability of CT, as well as the excellent soft tissue resolution of MRI, render these modalities particularly favorable for characterizing the continuity of intraluminal tumors. In the two cases in the present study, the IVLs appeared as soft masses in the right atrium and IVC on CT scans; the IVL completely occupied the IVC, iliac veins and internal iliac vein in a continuous manner in each case. A portion of the irregular uterine mass appeared to be anatomically connected to the tumor in the adjacent iliac veins. The IVLs of the two patients shared similar soft tissue density and enhanced features on CT or MRI scans with those reported in previous studies $(40,47)$. Of the 52 cases identified in the literature, $45(86.5 \%)$ underwent a contrast-enhanced CT scan or CT angiography. Heterogeneous enhancement of the tumors was observed in $27(60.0 \%)$ cases, consistent with the present cases, with mildly homogeneous or no enhancement in 13 (28.9\%) cases and homogeneous enhancement in $5(11.1 \%)$ cases.

The signal intensity of the lesions on MRI depends on the number of smooth muscle cells and vessels containing hyalinized fibrous tissue (48). The tumor in case 1 of the present study was mildly hyperintense on T1WI and T2WI, whereas the tumor in case 2 had a low to inter- 


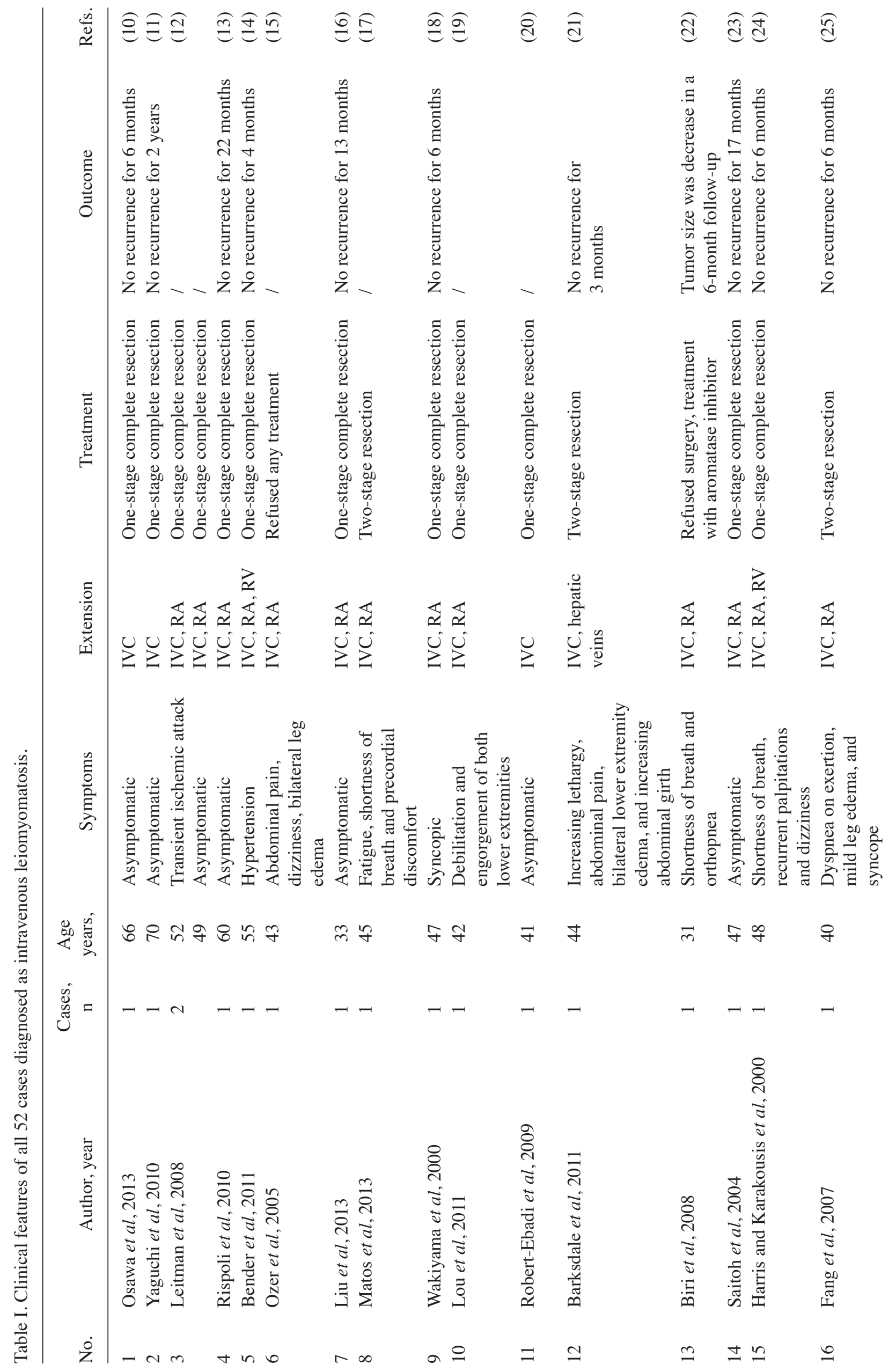




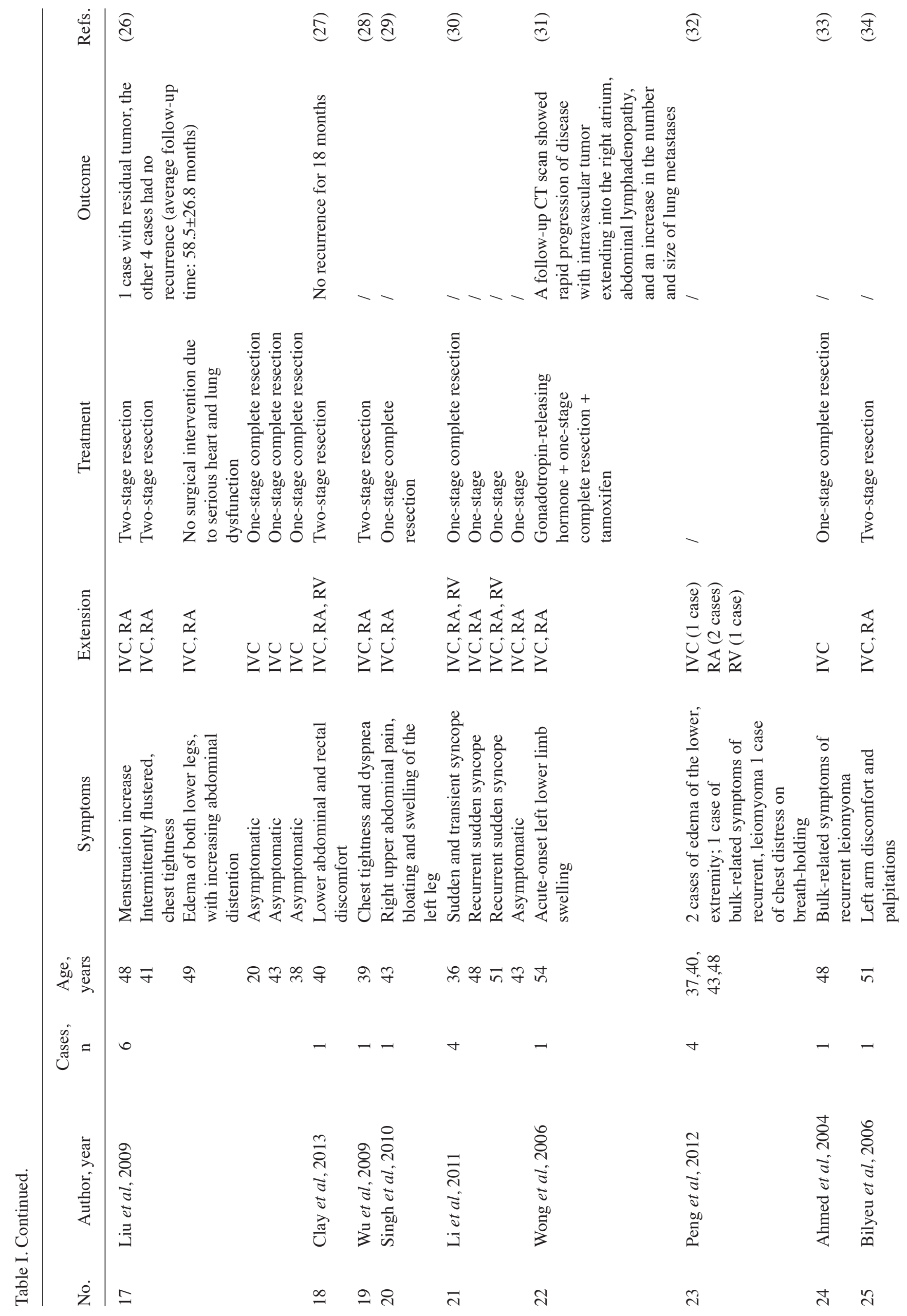




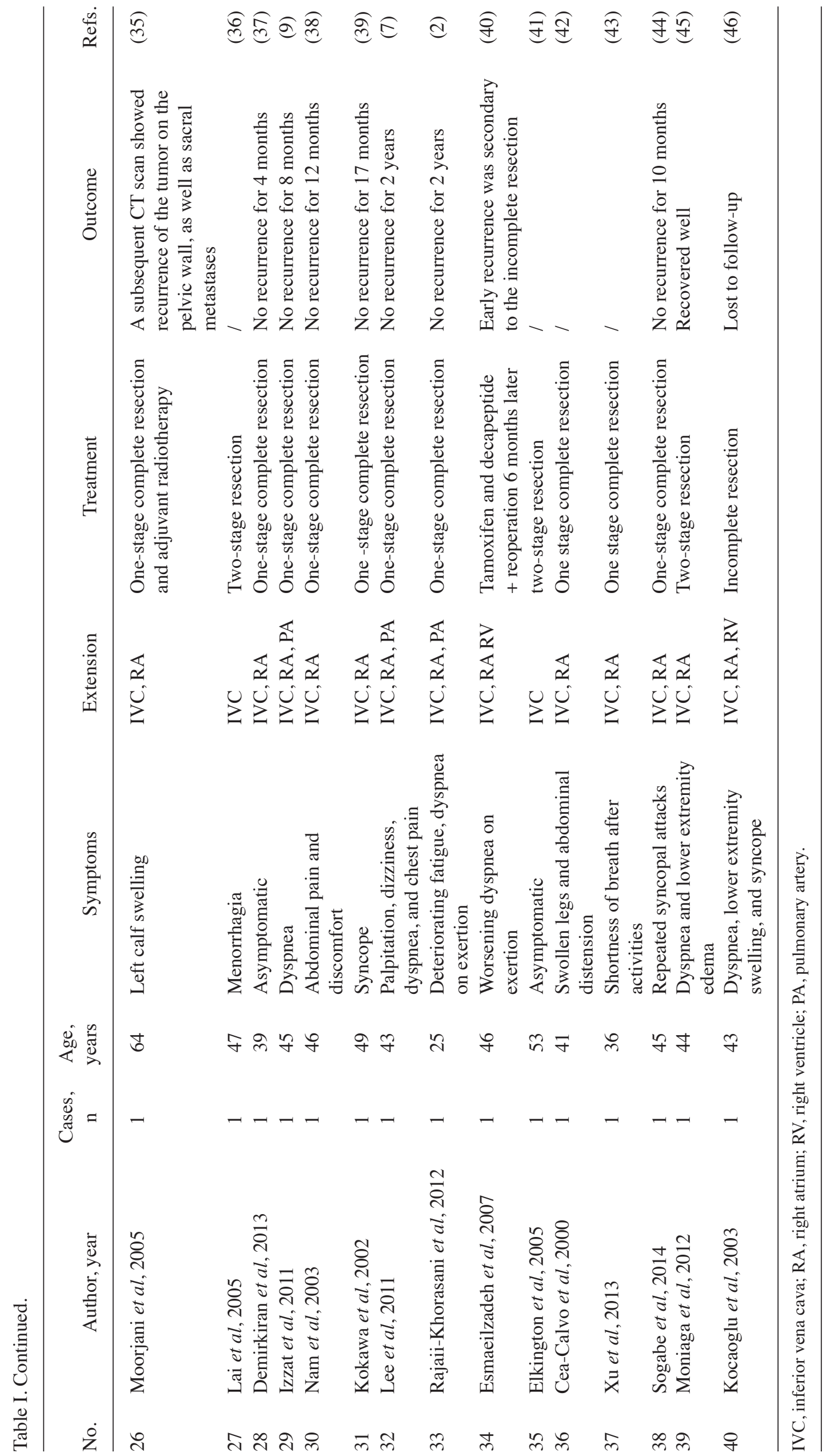


mediate signal intensity on T1WI and heterogeneous hyperintensity on T2WI; both tumors exhibited heterogeneous enhancement. There are conflicting reports with regards to the signal intensity of IVL on MRI scans; in certain studies, IVL presents as iso- to mildly hyperintense $(46,48)$, while in others it presents as hypointense $(49,50)$ on T1WI. The predominant findings on T2WI are heterogeneous-homogeneous hyperintense signals (46); however, Fasih et al (48) reported low signal intensity and Kang et al (49) reported isointensity to the myocardium. Of the 24 reported cases that underwent contrast-enhanced MRI scans, the intraluminal tumors exhibited mild heterogeneous enhancement in $45.8 \%$ cases, mild homogeneous enhancement in $29.2 \%$ and marked enhancement in $25.0 \%$, while the two present cases showed mild heterogeneous enhancement. We propose that the diverse pattern of phase-based enhancement may result from the nourishing vessels of the tumor originating from the pelvic vasculature.

The differential diagnosis of IVL predominantly includes thrombi within systemic veins, leiomyosarcoma arising from the wall of the IVC, right atrial myxoma and malignant thrombosis with carcinoma (34), as these entities have similar CT or MRI characteristics to IVL. However, intravenous thrombi show no enhancement following contrast agent administration due to their lack of vascular supply. Distinguishing between IVL and leiomyosarcoma of the IVC is challenging in the early stages of the disease, however, when the latter presents with visible infiltration and invasion around structures, diagnosis becomes more simple (51). Leiomyosarcoma arises from the vascular wall, and a gap between the tumor and vascular wall may not be visible on cross-sectional CT images from a certain point of view. Furthermore, current research indicates that IVL only occurs in women (52). Right atrial myxoma typically involves the cardiac chamber and does not normally extend into the IVC. The features of malignant thrombosis are similar to those of the primary tumor and the lesion is generally considerably smaller than the IVL. In addition, malignant thrombosis is not associated with a history of uterine myoma or surgery for uterine myoma (10). Complete surgical excision of the tumor is an appropriate treatment option for IVL, and the final diagnosis is dependent on histopathological analysis.

In conclusion, IVL should be considered upon presentation of a soft mass in the systemic veins, with or without extension to the right cardiac chamber, in female patients, particularly in patients with a history of uterine myoma. Furthermore, imaging modalities able to display the precise location and full-scale extension path of the tumor, such as CT and MRI, are of great importance in the presurgical assessment of IVL.

\section{References}

1. Norris HJ and Parmley T: Mesenchymal tumors of the uterus. V. Intravenous leiomyomatosis. A clinical and pathologic study of 14 cases. Cancer 36: 2164-2178, 1975.

2. Rajaii-Khorasani A, Kahrom M, Hashemzadeh M, Tayebi S, Ghazi M and Hamedanchi A: Pulmonary artery extension of uterine leiomyoma. J Card Surg 27: 466-469, 2012.

3. Dalainas I: Vascular smooth muscle tumors: Review of the literature. Int J Surg 6: 157-163, 2008.

4. Birch-Hirschfeld FV: Textbook of Pathological Anatomy. 5th edition. F.C.W. Vogel, Leipzig, pp226-258, 1896 (In German)
5. Durck H: Ueber ien kontinvierlich durch die entere holhlvene in das herz vorwachsendes: Fibromyom des uterus. Munch Med Wochenschr 54: 1154, 1907 (In German).

6. Borland DS and Wotring JW: Intravenous leiomyomatosis of the uterus and broad ligament: Report of a case. Am J Clin Pathol 42: 182-188, 1964.

7. Lee S, Kim DK, Narm KS and Cho SH: Pulmonary artery embolization of intravenous leiomyomatosis extending into the right atrium. Korean J Thorac Cardiovasc Surg 44: 243-246, 2011

8. Izzat MB, Bayazid S and Shuaibi I: Pelvic intravenous leiomyomatosis with trans-caval extension to the heart and pulmonary arteries. J Card Surg 26: 630-631, 2011.

9. Fukuyama A, Yokoyama Y, Futagami M, Shigeto T, Wada R and Mizunuma H: A case of uterine leiomyoma with intravenous leiomyomatosis-histological investigation of the pathological condition. Pathol Oncol Res 17: 171-174, 2011.

10. Osawa H, Hosaka S, Akashi O, Furukawa H and Egi K: A case of intravenous leiomyomatosis of uterine origin, extending through the inferior vena cava to right atrium. Gen Thorac Cardiovasc Surg 61: 104-107, 2013.

11. Yaguchi C, Oi H, Kobayashi H, Miura K and Kanayama N: A case of intravenous leiomyomatosis with high levels of hyaluronan. J Obstet Gynaecol Res 36: 454-458, 2010.

12. Leitman M, Kuperstein R, Medalion B, Stamler A, Porat E, Rosenblatt S, Konen E, Krakover R and Vered Z: A highly unusual right atrial mass presented in two women. Eur J Echocardiogr 9: 833-834, 2008

13. Rispoli P, Santovito D, Tallia C, Varetto G, Conforti M and Rinaldi M: A one-stage approach to the treatment of intravenous leiomyomatosis extending to the right heart. J Vasc Surg 52: 212-215, 2010.

14. Bender LC, Mitsumori LM, Lloyd KA and Stambaugh LE III: AIRP best cases in radiologic-pathologic correlation: Intravenous leiomyomatosis. Radiographics 31: 1053-1058, 2011.

15. Ozer N, Engin H, Akgül E, Sahiner L, Atalar E, Aksöyek S, Ovünç K, Ozmen F and Kes S: An unusual case of recurrent mass in the right atrium: Intravenous leiomyomatosis. Echocardiography 22: 514-516, 2005.

16. Liu W, Liu M and Xue J: Detection of intravenous leiomyomatosis with intracardiac extension by ultrasonography: A case report. Oncol Lett 6: 336-338, 2013.

17. Matos AP, Ramalho M, Palas J and Herédia V: Heart extension of an intravenous leiomyomatosis. Clin Imaging 37: 369-373, 2013.

18. Wakiyama H, Sugimoto T, Ataka K, Yamashita C, Tsuji Y, Nakagiri K, Inoue K and Okada M: Intravenous leiomyomatosis extending into the right ventricular cavity: One-stage radical operation using cardiopulmonary bypass - a case report. Angiology 51: 505-509, 2000.

19. Lou YF, Shi XP and Song ZZ: Intravenous leiomyomatosis of the uterus with extension to the right heart. Cardiovasc Ultrasound 9: $25,2011$.

20. Robert-Ebadi H, Terraz S, Mach N, Dubuisson JB, Kalangos A and Bounameaux $\mathrm{H}$ : Intravenous leiomyomatosis of the uterus: Link with new fertilisation methods? Swiss Med Wkly 139: 436, 2009.

21. Barksdale J, Abolhoda A and Saremi F: Intravenous leiomyomatosis presenting as acute Budd-Chiari syndrome. J Vasc Surg 54: 860-863, 2011.

22. Biri A, Korucuoglu U, Zumrutbas N, Tiras B and Guner H: Intravenous leiomyomatosis treated with aromatase inhibitor therapy. Int J Gynaecol Obstet 1013: 299-300, 2008.

23. Saitoh M, Hayasaka T, Nakahara K, Ohmichi M, Shimazaki Y and Kurachi H: Intravenous leiomyomatosis with cardiac extension. Gynecol Obstet Invest 58: 168-170, 2004.

24. Harris LM and Karakousis CP: Intravenous leiomyomatosis with cardiac extension: Tumor thrombectomy through an abdominal approach. J Vasc Surg 31: 1046-1051, 2000.

25. Fang BR, Ng YT and Yeh $\mathrm{CH}$ : Intravenous leiomyomatosis with extension to the heart: Echocardiographic features: A case report. Angiology 58: 376-379, 2007.

26. Liu B, Liu C, Guan H, Li Y, Song X, Shen K and Miao Q: Intravenous leiomyomatosis with inferior vena cava and heart extension. J Vasc Surg 50: 897-902, 2009.

27. Clay TD, Dimitriou J, McNally OM, Russell PA, Newcomb AE and Wilson AM: Intravenous leiomyomatosis with intracardiac extension - a review of diagnosis and management with an illustrative case. Surg Oncol 22: e44-e52, 2013.

28. Wu CK, Luo JL, Yang CY, Huang YT, Wu XM, Cheng CL, Chiang FT and Tseng CD: Intravenous leiomyomatosis with intracardiac extension. Inter Med 48: 997-1001, 2009. 
29. Singh T, Lamont PM, Otton GR and Thomson DS: Intravenous leiomyomatosis with intracardiac extension: First reported case in Australia. Heart Lung Circ 19: 50-52, 2010.

30. Li YQ, Mei F, Yang C, Lv P, Ouyang C and Jin B: Intravenous leiomyomatosis with right heart involvement - a report of 4 cases and literature review. J Huazhong Univ Sci Technolog Med Sci 31: 586-588, 2011.

31. Wong YY, Chu WC and Lam WW: Intravenous leiomyomatosis: Computed tomography diagnosis. Hong Kong Med J 12: 239-240, 2006.

32. Peng HJ, Zhao B, Yao QW, Qi HT, Xu ZD and Liu C: Intravenous leiomyomatosis: CT findings. Abdom Imaging 37: 628-631, 2012

33. Ahmed M, Zangos S, Bechstein WO and Vogl TJ: Intravenous leiomyomatosis. Eur Radiol 14: 1316-1317, 2004.

34. Bilyeu SP, Bilyeu JD and Parthasarathy R: Intravenous lipoleiomyomatosis. Clin Imaging 30: 361-364, 2006.

35. Moorjani N, Kuo J, Ashley S and Hughes G: Intravenous uterine leiomyosarcomatosis with intracardial extension. J Card Surg 20 382-385, 2005

36. Lai TK, Huang HY, Chan RY, Chin AC, Wong WC, Sit CY, Chan LK, Chung TK, Chu WS, Lo CF and Ng TW: Magnetic resonance venogram of intravenous leiomyomatosis. Hong Kong Med J 11: 524-526, 2005.

37. Demirkiran F, Sal V, Kaya U, Alhan C and Tokgozoglu N: Intravenous leiomyoma with extension to the heart: A case report and review of the literature. Case Rep Obstet Gynecol 2013: 602407, 2013.

38. Nam MS, Jeon MJ, Kim YT, Kim JW, Park KH and Hong YS Pelvic leiomyomatosis with intracaval and intracardiac extension: A case report and review of the literature. Gynecol Oncol 89: $175-180,2003$

39. Kokawa K, Yamoto M, Yata C, Mabuchi Y and Umesaki N: Postmenopausal intravenous leiomyomatosis with high levels of estradiol and estrogen receptor. Obstet Gynecol 100: 1124-1126, 2002.

40. Esmaeilzadeh M, Tavakolli A and Safaei A: Recurrent intracardiac leiomyomatosis. Can J Cardiol 23: 1085-1086, 2007.

41. Elkington NM and Carlton M: Recurrent intravenous leiomyomatosis with extension up the inferior vena cava. Aust N Z J Obstet Gynaecol 45: 167, 2005.
42. Cea-Calvo L, , Lozano F, Pombo M, Serrano A, Rodríguez E, Porto J, Pozuelo A and González C: Images in cardiovascular medicine. Uterine intravenous leiomyomatosis extending through the inferior vena cava into the right cardiac cavities. Circulation 101: 581-583, 2000.

43. Xu ZF, Yong F, Chen YY and Pan AZ: Uterine intravenous leiomyomatosis with cardiac extension: Imaging characteristics and literature review. World J Clin Oncol 4: 25-28, 2013.

44. Sogabe M, Kawahito K, Aizawa K, Sato H and Misawa Y: Uterine intravenous leiomyomatosis with right ventricular extension. Ann Thorac Cardiovasc Surg 20 (Suppl): 933-936, 2014.

45. Moniaga NC and Randall LM: Uterine leiomyomatosis with intracaval and intracardiac extension. Gynecol Oncol Rep 2: 130-132, 2012

46. Kocaoglu M, Bulakbasi N, Ugurel MS, Ors F, Tayfun C and Ucoz T: Value of magnetic resonance imaging in the depiction of intravenous leiomyomatosis extending into the heart. J Comput Assist Tomogr 27: 630-633, 2003.

47. Kawakami S, Sagoh T, Kumada H, Kimoto T, Togashi K, Nishimura K, Honda H, Yamada M and Noguchi M: Intravenous leiomyomatosis of uterus: MR appearance. J Comput Assist Tomogr 15: 686-689, 1991.

48. Fasih N, Prasad Shanbhogue AK, Macdonald DB, Fraser-Hill MA, Papadatos D, Kielar AZ, Doherty GP, Walsh C, McInnes M and Atri M: Leiomyomas beyond the uterus: Unusual locations, rare manifestations. Radiographics 28: 1931-1948, 2008.

49. Kang LQ, Zhang B, Liu BG and Liu FH: Diagnosis of intravenous leiomyomatosis extending to heart with emphasis on magnetic resonance imaging. Chin Med J (Engl) 125: 33-37, 2012.

50. Cohen DT, Oliva E, Hahn PF, Fuller AF Jr and Lee SI: Uterine smooth-muscle tumors with unusual growth patterns: Imaging with pathologic correlation. AJR Am J Roentgenol 188: 246-255, 2007.

51. McDonaldDK,Kalva SP,Fan CM and Vasilyev A: Leiomyosarcoma of the uterus with intravascular tumor extension and pulmonary tumor embolism. Cardiovasc Intervent Radiol 30: 140-142, 2007.

52. Huang J, Liu G, Lu JP, Wang F, Wang L and Jin AG: Primary intraluminal leiomyosarcoma of the inferior vena cava: Value of MRI with contrast-enhanced MR venography in diagnosis and treatment. Abdom Imaging 36: 337-341, 2011. 